\title{
MIMO-SAR AND THE ORTHOGONALITY CONFUSION
}

\author{
G. Krieger, M. Younis, S. Huber, F. Bordoni, A. Patyuchenko, J. Kim, P. Laskowski, \\ M. Villano, T. Rommel, P. Lopez-Dekker, and A. Moreira
}

Microwaves and Radar Institute, German Aerospace Center (DLR), Oberpfaffenhofen, Germany

\begin{abstract}
This paper reviews radar architectures that employ multiple transmit and multiple receive channels to improve the performance of synthetic aperture radar (SAR) systems. These advanced architectures have been dubbed multiple-input multiple-output SAR (MIMO-SAR) in analogy to MIMO communication systems. Considerable confusion arose, however, with regard to the selection of suitable waveforms for the simultaneous transmission via multiple antennas. In this paper, it is shown that the mere use of orthogonal waveforms is insufficient for the desired performance improvement in view of most SAR applications. As a solution to this fundamental MIMO-SAR problem we had previously suggested to exploit the special data acquisition geometry of a side-looking imaging radar equipped with multiple receiver channels in addition to appropriately designed waveforms transmitted by multiple antennas. Here, we extend this approach to a more general set of radar waveforms with special correlation properties that satisfy a short-term shift-orthogonality condition. We show that the echoes from simultaneously transmitted pulses can be separated if the short-term shift orthogonality is combined with digital beamforming on receive in elevation. This enables the implementation of a fully functional MIMO-SAR without correlation noise leakage for extended scattering scenarios.
\end{abstract}

Index Terms: Multiple-Input Multiple-Output Synthetic Aperture Radar (MIMO-SAR), Multidimensional Waveform Encoding, Digital Beamforming (DBF), Orthogonal Frequency-Division Multiplexing (OFDM).

\section{MOTIVATION}

Synthetic Aperture Radar (SAR) is a powerful remote sensing technique that can provide high resolution radar images of the terrestrial surface independent of weather and sunlight illumination. This capability makes SAR an ideal candidate for a global Earth observatory that continuously monitors the plethora of natural and anthropogenic processes which permanently restructure the surface of our planet. The mapping capabilities of current SAR sensors are, however, insufficient to reach this ambitious goal with a reasonable number of satellites. Next generation SAR missions like Tandem-L will employ advanced digital beamforming techniques to improve the imaging performance by more than one order of magnitude if compared to state of the art sensors like TerraSAR-X [1]. In the future, further improvements can be achieved by combining digital beamforming on receive with multiple transmit channels radiating appropriately designed waveforms [2]. These enhanced architectures, now dubbed MIMO-SAR, offer not only the opportunity to map wider image swaths with improved spatial resolution, but they enable also novel SAR imaging modes that are well suited to mitigate or even resolve hitherto contradicting user requirements.

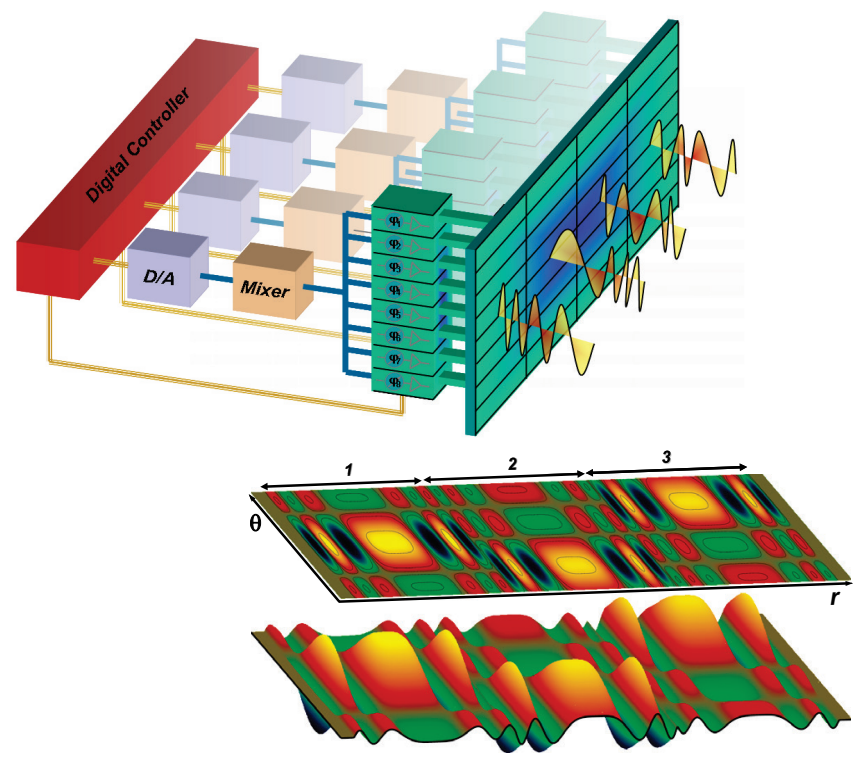

Figure 1: A MIMO-SAR architecture employs in addition to multiple receivers multiple transmit channels (top) to generate waveforms that are non-separable with regard to their spacetime coordinates (bottom). Conventional SAR systems are in contrast characterized by transmitting waveforms (or phase fronts) that can be written as the product of a time-only modulation function and a space-only angular antenna pattern (images from [9]).

\section{EARLY DEVELOPMENTS TOWARS MIMO-SAR}

A receiver with $N_{R x}$ apertures allows a simultaneous sampling of the arriving wavefronts with $N_{R x}$ effective phase centers. The number of effective phase centers can be increased by using not only one but multiple transmitters. If $N_{T x}$ transmitters are employed in addition to $N_{R x}$ receivers, the total number of possible phase centers increases from $N_{D B F}=N_{R x}$ to $N_{M I M O}=N_{T x} \cdot N_{R x}$, where the virtual phase center locations can be computed from a spatial convolution of the physical transmit and receive antenna positions [3]. Inspired by this diversity gain, reference [4] investigated the advantages of such a MIMO architecture to mitigate RCS scintillations and the edited collection [5] compiled further analyses of MIMO concepts and processing techniques in the context of non-imaging radar systems. With regard to real aperture radar imaging, additional transmit antennas were suggested to improve the angular resolution and to add interferometric/tomographic capabilities in the context of a forward looking imaging radar experiment [6]. A similar approach was later elaborated in more detail to improve the cross-track resolution of a 3-D radar system [7]. 
Regarding SAR imaging, first suggestions of using a MIMO-SAR architecture emerged in May 2006 in [8], [9] (cf. Figure 1). In these publications it was shown that the possible benefits of using multiple transmit channels together with a multi-channel receiver range from an increased spatial coverage and geometric resolution to the improved suppression of range and azimuth ambiguities to the provision of additional baselines for GMTI and interferometric applications. In [10], and later again in [11], the possible application range was further extended to super-resolution. Another interesting opportunity of MIMOSAR is fully-polarimetric imaging without a doubling of the PRF [12], [13]. For this, it was suggested to transmit two orthogonally polarized waves within the same radar pulse.

\section{ORTHOGONAL WAVEFORMS: THE GREAT CONFUSION}

To fully exploit the increased spatial diversity provided by additional transmit antennas, it is necessary to separate the radar echoes from the multiple transmit signals within the receiver. To this end, several authors suggested to transmit mutually orthogonal waveforms. Notable confusion arose, however, what exactly is meant by "orthogonal".

Some publications (cf., e.g., [14], [15], [16]) just require

$$
\int s_{i}^{*}(t) \cdot s_{j}(t) \cdot d t=0 \text { if } i \neq j
$$

where $s_{i}(t)$ and $s_{j}(t)$ are the transmitted signals from any two different apertures/channels. While this condition allows perfect separation of the scattered waveforms from a single point target, it is not sufficient for signal separation in an extended scattering scenario [2]. The reason for the poor performance is that the orthogonality is not ensured for arbitrary shifts between the transmit signals. As a result, the energy from spatially separated scatterers illuminated by the other waveform(s) does not vanish after range focusing but appears either smeared or at different positions [2]. This reasoning is also evident by considering range focusing as an all-pass filter with a "matched" phase function in the frequency domain. As long as all orthogonal signals share the same frequency band, a mismatch of the phase will not affect the magnitude of the spectrum. According to Parseval's theorem, the output signals of all focusing filters will hence have the same power/energy regardless of whether a matched or an unmatched signal is present. Depending on the number of transmitters, the smeared energy from the orthogonally illuminated scatterer ensemble may hence even exceed the focused target response.

To avoid this inherent problem, some other publications (cf., e.g., [10], [17], [18]) require the orthogonality for arbitrary time shifts $\tau$ between the signals $s_{i}(t)$ and $s_{j}(t)$

$$
\int s_{i}^{*}(t) \cdot s_{j}(t+\tau) \cdot d t=0 \quad \forall \tau \in \mathbb{R}, i \neq j
$$

This enables a perfect signal separation also in case of a distributed scatterer scenario (possible effects from Doppler

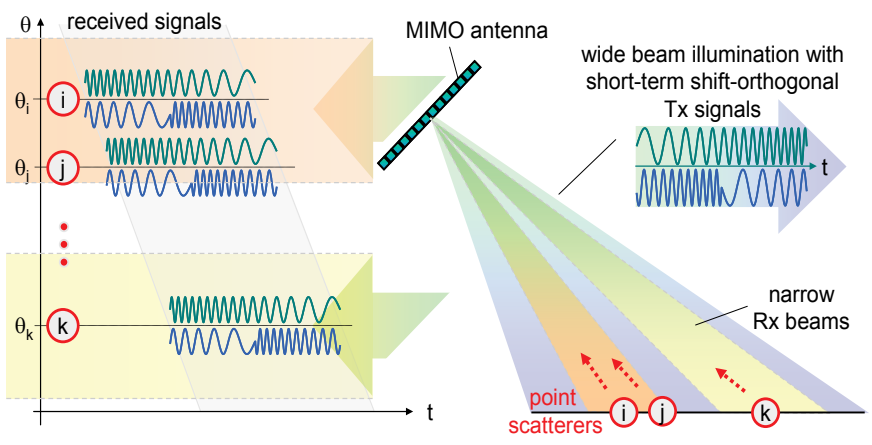

Figure 2: MIMO-SAR based on two short-term shiftorthogonal transmit signals. The waveforms are designed such that their cross-correlation vanishes for small time offsets ( $i$ and $\mathbf{j}$ ). For larger time offsets, the otherwise correlated signals are separated via narrow $R x$ beams ( $i$ and $k$ ). This exploitation of the imaging geometry together with appropriately designed transmit waveforms enables the simultaneous use of multiple Tx signals and the unambiguous separation of their radar echoes also in case of a distributed scatterer scenario.

shifts will be discussed later). However, an immediate consequence of this requirement is that $s_{i}(t)$ and $s_{j}(t)$ must have nonoverlapping spectral support. This is immediately evident from the cross-correlation theorem

$$
s_{i}(t) \otimes s_{j}(t)=\mathcal{F}^{-1}\left[S_{i}^{*}(f) \cdot S_{j}(f)\right]
$$

where $\otimes$ is a short hand for the cross-correlation integral of Eq. (2), $\mathcal{F}^{-1}$ denotes the inverse Fourier transform and $S_{i}(f)$ and $S_{j}(f)$, are the Fourier transforms of $s_{i}(t)$ and $s_{j}(t)$, respectively. This theorem is similar to the well-known convolution theorem and states that the cross-correlation of two functions can be expressed (up to a sign change in the conjugate complex function) in the frequency domain as the product of their individual Fourier transforms.

It is evident from Eq. (3) that Eq. (2) can only be fulfilled if the product between $S_{i}(f)$ and $S_{j}(f)$ vanishes for all $f$. This implies that $S_{i}(f)$ and $S_{j}(f)$ have no common spectral support, making them of limited use if a coherent combination of the radar echoes from the orthogonal waveforms is desired, as it is necessary for all MIMO-SAR applications mentioned in Sect. 2 .

\section{THE SOLUTION: DIGITAL BEAMFORMING}

To overcome the fundamental challenges described in the previous section, we suggested in [8], [9], [2] to employ especially designed waveforms together with digital beamforming on receive. The basic idea can be expressed by a kind of restricted shift-orthogonality condition

$$
\int h(\tau) \cdot s_{i}^{*}(t) \cdot s_{j}(t+\tau) \cdot d t=0
$$

where in comparison to Eq. (2) an additional weighting function $h(\tau)$ has been included which depends on the relative time shift $\tau .{ }^{1}$ The function $h(\tau)$ is a consequence

\footnotetext{
${ }^{1}$ a possible dependency of $h(\tau)$ on $t$ is neglected to ease understanding.
} 


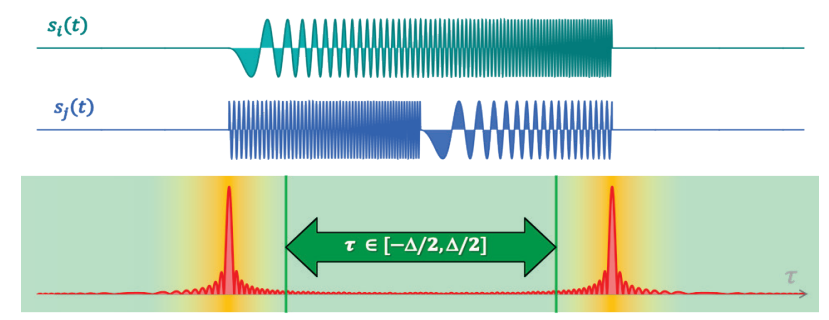

Figure 3: Illustration of short-term shift orthogonality. The two waveforms $s_{i}(t)$ and $s_{j}(t)$ cover the same frequency band and are orthogonal for mutual time shifts within the interval $\tau \in[-\Delta / 2, \Delta / 2] . s_{i}(t)$ and $s_{j}(t)$ satisfy hence the restricted shiftorthogonality condition from Eq. (4) for $h(\tau)=\operatorname{rect}(\tau / \Delta)$.

from the side looking geometry of our ground imaging radar. To understand this, note that there exists a direct correspondence between the angle of arrival and the radar signal delay. This means that signals from targets at different ranges arrive also from different look angles. As a result, the echoes from targets with sufficient mutual range delay $\tau$ can be separated in a multi-channel receiver by an appropriate beamforming in the elevation direction. This is illustrated in Figure 2, which shows on the lower right a set of point scatterers that are illuminated by two orthogonal $\mathrm{Tx}$ signals satisfying the restricted shift-orthogonality condition from Eq. (4). The radar echoes from the individual point scatterers arrive then according to the different pulse travel times at different instances of time as shown in the upper left of Figure 2, where the pulse echoes from the neighboring scatterers $i$ and $j$ arrive significantly before those from the more distant scatterer k. Digital beamforming on receive enables a reliable separation (or spatial filtering) of the radar echoes $\{i, j\}$ from those of the point scatterer $k$ as illustrated by the yellow and orange beams in Figure 2 . For this, a sufficient number of receiver channels should be available and the antenna height should exceed the value given in Eq. (14) of [2]. For small antenna heights, moreover, an accurate null steering may be required.

On the other hand, the echoes from $\mathrm{i}$ and $\mathrm{j}$ cannot be mutually separated by the spatial beamforming since the scatterers are located too close together. The only means to avoid a mutual interference of the radar echoes for these closely spaced scatterers is an appropriate choice of the transmitted waveforms such that they are mutually orthogonal within the time interval that cannot be resolved by the spatial beamforming. As an example, consider in Eq. (4) the function $h(\tau)=\operatorname{rect}(\tau / \Delta)$, which is one within the interval $[-\Delta / 2, \Delta / 2]$ and zero elsewhere. We hence must only ensure that the left hand side of Eq. (2) vanishes for shifts $\tau$ that are smaller than $\Delta / 2$. This offers a new degree of freedom to re-introduce spectral overlap between $S_{i}(f)$ and $S_{j}(f)$. One possibility would be to use mutually shifted chirp pulses as suggested in [8], [9], [2]. A similar approach is illustrated in Figure 3 which employs a signal with linear frequency modulation for $s_{i}(t)$ and a second signal $s_{j}(t)$ with a sufficient offset in its instantaneous frequency. This offset can e.g. be obtained by a cyclic shift of the chirped signal $s_{j}(t)$. The special choice for $s_{i}(t)$ and $s_{j}(t)$ ensures that there is no interference between the signal returns for closely separated scatterers, while scatterers with a larger mutual separation would cause an interference between the received signals. ${ }^{2}$ This interference can, however, be avoided by the spatial filtering via digital beamforming on receive as illustrated in Figure 2.

To summarize, the combination of appropriately designed short-term shift-orthogonal $\mathrm{Tx}$ waveforms with digital beamforming on receive in elevation enables hence the implementation of a MIMO-SAR not only for an academic scenario consisting of one or a few closely spaced scatterers as considered in several recent MIMO-SAR publications (cf., e.g., [15], [16]), but also for a realistic spaceborne SAR scenario where the swath width exceeds the spatial extension of the transmitted pulse.

\section{OFDM: AN ALTERNATIVE?}

Recently, it was suggested to employ orthogonal frequency division multiplexing (OFDM) techniques to obtain a pair of transmit signals that satisfy Eq. (2). For this, the bandwidth of two discrete and time limited chirp signals was divided in the frequency domain by assigning subsequent frequencies to either one or the other waveform [13]. This results in a comb-like spectrum for each Tx signal and no spectral overlap between the two waveforms. The scene is therefore mapped by a pair of orthogonal waveforms with mutually exclusive spectral support.

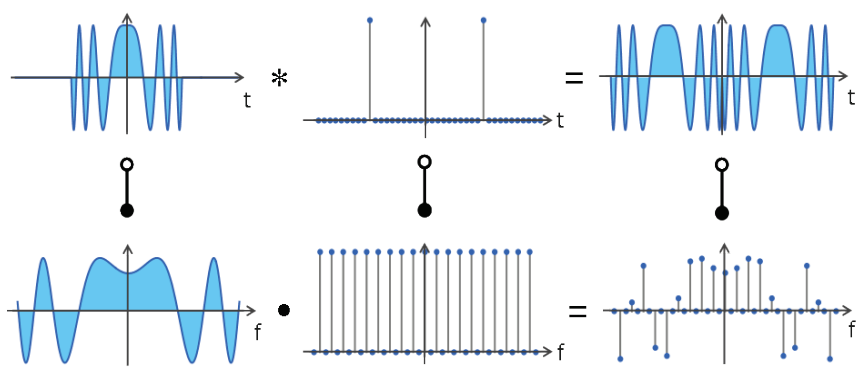

Figure 4: OFDM waveform generation in the frequency (bottom) and time (top) domain. The comb-like spectrum corresponds in the time domain to two repeated sub-pulses.

To understand the implications of this special kind of SAR data acquisition, it is instructive to take a closer look at the transmitted waveforms. Figure 4 shows that the comb-like OFDM spectrum corresponds in the time domain to a signal that is composed of two sub-pulses. The pulse repetition is a direct consequence of the comb-like spectrum and independent of the original waveform. This repeated subpulse structure implies in turn that the focused image from

\footnotetext{
${ }^{2}$ This special choice of waveforms provides moreover maximum Doppler tolerance, avoiding for example the problem from Sect. 5 that a portion of the signal energy from one channel may be leaked into the other channel if a broad Doppler spectrum is acquired.
} 
each waveform becomes ambiguous in range if the scene extension is longer than half the OFDM pulse length. The mere use of OFDM Tx signals together with an appropriate matched filter (or any other OFDM processing) is therefore insufficient for an unambiguous wide-swath MIMO-SAR imaging! The ambiguity can only be resolved if additional information is provided by employing multiple receive channels. The extra information can then be used to suppress the range ambiguity by digital beamforming on receive as illustrated in Figure 5. It should hence be clear, that the suggested OFDM-MIMO-SAR requires the same basic principle as it was originally suggested by the concept of multidimensional waveform encoding (cf. Section 4). Only for scenes that are shorter than half of the transmitted pulse length, one may neglect the range ambiguity suppression by digital beamforming on receive. This may be a viable solution in a bistatic automotive radar [19] or the ISAR system in [16] where the range from which radar echoes are received above noise level is shorter than the length of the transmitted pulse.

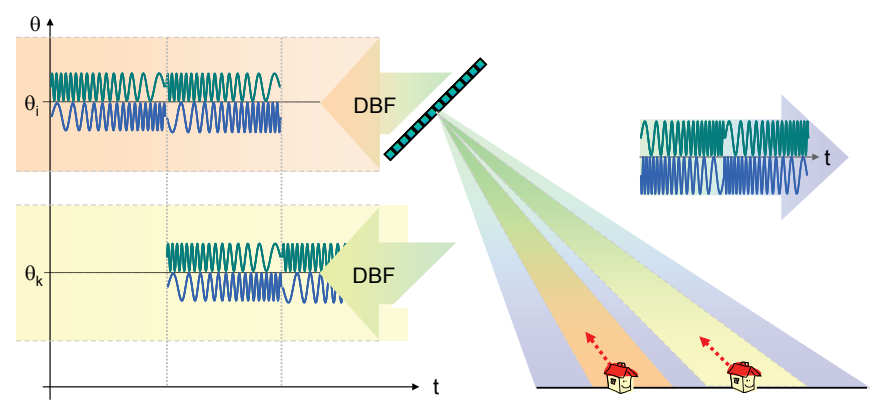

Figure 5: The use of OFDM signals results in range ambiguities that must be resolved by digital beamforming on receive.

After pointing out the similarities between OFDM and multidimensional waveform encoding, the question remains which kind of waveform encoding is the most appropriate. For this, one should be aware that in the suggested OFDM modulation a possible issue arises from the close separation of the frequency components assigned to either one or the other waveform. If, for example, a $100 \mu$ s long pulse is employed, the frequency components will be separated by only $10 \mathrm{kHz}$. Such a separation is, however, already close to the Doppler bandwidth of a high-resolution wide-swath SAR system with $1 \mathrm{~m}$ azimuth resolution. It is therefore inevitable that a notable portion of the Doppler spectrum is leaked into the "orthogonal" channel, thereby deteriorating the image quality. In contrast, the instantaneous frequency bands of the waveforms from Figure 3 are typically separated by several $10 \mathrm{MHz}$ and Doppler leakage is therefore not an issue at all. Another question concerns the required antenna height to suppress the range ambiguities in the different approaches. Here, a short analysis reveals that the same minimum antenna height as provided in Eq. (14) of [2] has to be used for both OFDM and the suggested short-term shift-orthogonal waveform examples provided in this paper.

\section{CONCLUSIONS}

MIMO-SAR employs multiple transmit and receive channels to improve the imaging performance and to acquire novel interferometric and tomographic information products with a minimum number of antenna elements. In selected airborne scenarios it may be sufficient to transmit the different $\mathrm{Tx}$ signals in subsequent pulse repetition intervals. Such a strategy is, however, in general not applicable to a spaceborne scenario where the high sensor velocity poses notable ambiguity constraints. A prominent example is the reduced unambiguous swath width in the alternating bistatic mode of TanDEM-X which can be regarded as a MIMO-SAR with two transmit and two receive channels that provide together three virtual phase centers. To overcome this fundamental MIMO-SAR challenge, we suggested to simultaneously transmit multiple waveforms that are mutually short-term shift-orthogonal. The lack of orthogonality for longer shifts is then resolved by digital beamforming on receive. This enables not only the acquisition of additional phase centers for novel SAR applications but also the implementation of a new class of hybrid and adaptive SAR modes [1], [2].

\section{REFERENCES}

[1] G Krieger, I Hajnsek, K Papathanassiou, M Younis, A Moreira. Interferometric Synthetic Aperture Radar (SAR) Missions Employing Formation Flying. Proceedings of the IEEE, vol. 98, pp. 816 - 843, 2010.

[2] G Krieger, N Gebert, A Moreira. Multidimensional Waveform Encoding: A New Digital Beamforming Technique for Synthetic Aperture Radar Remote Sensing. IEEE Trans. Geosc. Remote Sens., vol. 46, no.1, pp. 31-46, 2008.

[3] RC Heimiller, JE Belyea, PG Tomlinson, Distributed Array Radar, IEEE Transactions on Aerospace Electronic Systems, vol. 19, pp. 831-839, 1983.

[4] E Fishler et al., MIMO RADAR: An Idea Whose Time Has Come. IEEE Radar Conference, Philadelphia, PE, USA, April 2004.

[5] J Li, P Stoica. MIMO Radar Signal Processing. John Wiley and Sons, 2009.

[6] G. Krieger, J. Mittermayer, S. Buckreuß, M. Wendler, T. Sutor, F. Witte, A. Moreira. Sector Imaging Radar for Enhanced Vision. Aerospace Science and Technology, vol. 7, pp. $147-158,2003$.

[7] J Klare, A Brenner, J Ender. A new Airborne Radar for 3D Imaging - Image Formation using the ARTINO Principle. EUSAR, Dresden, Germany, 2006.

[8] G Krieger et al., patent appl. DE 102006022814, May 13, 2006.

[9] G Krieger, N Gebert, A Moreira. Digital Beamforming Techniques for Spaceborne Radar Remote Sensing. EUSAR, Dresden, Germany, 2006.

[10] J Ender. MIMO-SAR. Internat. Radar Symposium, Cologne, Germany, 2007.

[11] D Christallini, D Patina, P Lombardo. Exploiting MIMO SAR Potentialities with Efficient Cross-Track Constellation Configurations for Improved Range Resolution. IEEE Trans. Geosci. Remote Sens., vol. 49, pp. 38-52, 2011.

[12] G Krieger, N Gebert, A Moreira, Multidimensional Waveform Encoding for Synthetic Aperture Radar Remote Sensing. IET International Conference on Radar Systems, Edinburgh, UK, 2007.

[13] J Kim, M Younis, A Moreira, W Wiesbeck. A Novel OFDM Waveform for Fully Polarimetric SAR Data Acquisition. EUSAR, Aachen, Germany, 2010.

[14] WQ Wang. Virtual Antenna Array Analysis for MIMO Synthetic Aperture Radars. International Journal of Antennas and Propagation, 2012.

[15] WQ Wang, Space-Time Coding MIMO-OFDM SAR for High-Resolution Imaging, IEEE Trans. Geosci. Remote Sens., vol. 49, pp. 3094-3104, 2011.

[16] G Castellanos, M Jirousek, M Peichl. Orthogonal Waveform Experiments with a Highly Digitized Radar. EUSAR, Nuremberg, Germany, 2012.

[17] WQ Wang. Applications of MIMO Technique for Aerospace Remote Sensing. Proc. of Aerospace Remote Sensing, USA, 2007.

[18] J Klare. Digital Beamforming for a 3D MIMO SAR - Improvements Through Frequency and Waveform Diversity. IGARSS, Boston, MA, 2008.

[19] C Sturm, W Wiesbeck. Waveform Design and Signal Processing Aspects for Fusion of Wireless Communications and Radar Sensing. Proceedings of the IEEE, vol. 99, pp. 1236-1259, 2011. 